\title{
La medición del clima laboral
}

\author{
${ }^{1}$ Norma Placencia Galindo \\ ${ }^{1}$ Universidad Tecnológica Equinoccial, Quito - Ecuador, pgnf1030322@ute.edu.ec
}

Recepción/Received: 13, 10, 2013

Aceptación/Accepted: 26, 11, 2013

Publicado/Published: 20, 12, 2013

\section{Resumen:}

Para garantizar una buena calidad de la información recolectada en mediciones del clima laboral mediante cuestionarios, estos deben elaborarse utilizando normas técnicas establecidas para tal efecto, de manera que garanticemos la calidad de la información recolectada. Entre los aspectos que se deben considerar están la validez del instrumento de medición y la confiabilidad de los resultados obtenidos. Medir el clima organizacional significa medir varios factores relacionados con la percepción de las personas y sus constructos, así como acogerse a varias teorías organizacionales y psicológicas del comportamiento humano. Mientras estas actividades se lleven a cabo con criterio científico y de respeto a las personas, se verán mejores resultados en las mediciones.

\section{Palabras clave:}

Clima laboral, constructo, cuestionario, validez

\begin{abstract}
:
When measuring working environments using questionnaires, their preparation should be done following technical standards in order to guarantee the quality of the information collected. Among aspects to be considered are the validity of the measurement instrument and the reliability of the results. Measuring organizational climate means gauging several factors related to people's perception and their constructs which are covered by several organizational and psychological theories on human behavior. If these tasks are performed following scientific discipline and are met with respect towards people, the results are more likely to improve.
\end{abstract}

\section{Keywords:}

Working environment, construct, questionnaire, validity 


\section{INTRODUCCIÓN}

Las mediciones del clima laboral o clima organizacional están de moda en las empresas públicas y privadas, grandes y pequeñas, de producción o de servicios. Funcionarios y empleados de diversas profesiones y roles creen que puede realizar estas mediciones, generalmente mediante cuestionarios que aplicados a los empleados permitirán obtener un diagnóstico del clima laboral.

Los cuestionarios pueden encontrarse en publicaciones de variada índole, como revistas, prensa, artículos, libros y aún en Internet. Existen sitios web en donde es posible crear en minutos una encuesta del clima laboral, recopilar respuestas y hasta publicarlas en un blog, compartir los resultados en las redes sociales y analizar las respuestas con otros miembros de la red.

En las empresas y organizaciones, es frecuente el envío de los cuestionarios de medición del clima laboral vía intranet con la petición de contestar con la mayor sinceridad posible, garantizando la confidencialidad de la información; este último aspecto no siempre es posible como se habrán percatado quienes han contestado estos cuestionarios.

En la actualidad una gran cantidad de fallas, quiebres o conflictos organizaciones son atribuidos al clima laboral. En épocas cercanas, se analizaban la estructura, los procesos, los sistemas y subsistemas, la competencia, el ciclo de vida de los productos, la tecnología, la comunicación, las relaciones interpersonales, los estilos de dirección, los perfiles de competencias de los colaboradores, la satisfacción en el trabajo, la motivación, el empoderamiento, el sentido de pertenencia, etc. Ahora todos los problemas que aparecen en la organización son atribuidos al clima organizacional y la solución está en medir, generalmente con un cuestionario, cuya validez, confiabilidad, predicción y demás parámetros de calidad medianamente científica y ética, son profundamente cuestionables. A este afán de medir se añade el tema del objetivo de tal medición, que en la mayoría de ocasiones, se queda en el diagnóstico.

Una vez encontrados varios o ningún problema, los administradores no saben qué hacer con la información, que probablemente será olvidada hasta que los problemas de la empresa, que originaron la medición, vuelvan a escalar y a ser notorios. Esto se produce, en ocasiones, debido a que mientras se aplica la encuesta o cuestionario, existe una especie de válvula de escape originada en la comunicación informal de los empleados respecto de las preguntas que les hicieron en el documento que pretende medir el clima laboral.

Una de las situaciones en las cuales se aplican los cuestionarios de medición del clima laboral son aquellas que tienen como protagonista a un jefe cuyo estilo de dirección es el denominado autócrata o autoritario y no alcanza a entender el porqué del malestar de los empleados; también ocurre que se pretende explicar las fallas de la organización atribuyéndole al clima organizacional la solución y la causa de la mayor parte de los problemas que se suscitan en el trabajo.

\section{II. ¿QUÉ ES EL CLIMA LABORAL?}

Al revisar varios artículos y revistas encontramos que no llega a definirse el clima laboral y más bien nos hablan de productividad, satisfacción en el trabajo, compañerismo, ventaja competitiva, entre otros temas. Una definición que encontramos en revistas, estudios, investigaciones y hasta en la prensa escrita, sin que referencien el autor dice: el clima laboral es el ambiente humano y físico en el que se desarrolla el trabajo cotidiano, influye en la satisfacción personal y en la productividad.

Si analizamos esta definición se relaciona con los riesgos del trabajo, seguridad industrial y salud ocupacional, aunque en un contexto más amplio y holístico, todo influye e interactúa con todo.

Una definición interesante plantea Cotton (2013):

En la literatura de investigación sobre comportamiento organizacional, la noción de clima organizacional está definida en principio en términos de percepciones compartidas de las políticas organizacionales, prácticas $y$ procedimientos (e.j. Reichers \& Schneider, 1990). Además el clima organizacional incluye las percepciones de los empleados y evaluaciones de las prácticas de liderazgo, procesos de toma de decisiones, relaciones interpersonales entre empleados, papeles, roles, reconocimientos, 
apreciacionesyobjetivos. Elclima organizacional, refleja la forma en que están hechas las cosas en un entorno de trabajo determinado.

En su artículo, Carrie (2012) indica:

(...) En un comunicado, el jefe de personal de Google, Laslo Buck, dijo que el trabajo no debe ser una obligación impuesta por el dinero, sino un lugar agradable en el que las personas se sientan cómodas. "Sin duda los resultados se notan cuando el compromiso, la motivación y el estado de ánimo de las personas están en armonía con el trabajo", sostuvo. Porque contar con un ambiente con condiciones físicas, psicológicas y administrativas adecuadas o diseñadas especialmente para la conformidad de todos los integrantes de la empresa, pueden ser elementos clave para el posicionamiento de esta.

Al hablar de clima laboral, entonces, tenemos que referirnos necesariamente a por lo menos dos conceptos: ¿qué es el clima laboral? y ¿cuál es la percepción humana que se intenta medir con relación al clima laboral? Cuando se analizan estos dos conceptos nos damos cuenta de que también debemos hacer referencia, como mínimo, a una o varias teorías sobre la personalidad, las actitudes y la administración o dirección de empresas, sin olvidarnos de las técnicas de medición que utilizaremos.

Respecto de las percepciones, varias definiciones se encuentran en Guardiola (2002):

La percepción puede definirse como el conjunto de procesos y actividades relacionados con la estimulación que alcanza a los sentidos, mediante los cuales obtenemos información respecto de nuestro hábitat, las acciones que efectuamos con él y nuestros propios estados internos.

En el resumen del mismo documento, encontramos que se define a la percepción de la siguiente manera: "la percepción es la imagen mental que se forma con la ayuda de la experiencia y necesidades, resultado de un proceso de selección, organización e interpretación de sensaciones. La percepción de los individuos es subjetiva, selectiva y temporal".
Valbuena de la Fuente (1979) afirma: "La percepción es un fenómeno mucho más complejo y necesitamos un esquema que nos permita estudiar las situaciones presentes como dotadas de significados, sin adentrarnos en las configuraciones y predisposiciones de las personas".

Estos conceptos exponen la influencia recíproca de la persona y su medio ambiente físico, psicológico, social.

Al mencionar las percepciones como base para la medición del clima laboral, necesariamente tenemos que referirnos al sujeto en el cual sucede el proceso de la percepción.

Una de las teorías que menciona la influencia recíproca que existe entre la persona y el medio es la teoría de Allport, en la cual se manifiesta la influencia conjunta de la personalidad y de las situaciones. Allport define a la personalidad de la siguiente manera: "Personalidad es la organización dinámica, dentro del individuo, de los sistemas psicofísicos que determinan sus ajustes únicos al ambiente".

Otras definiciones citadas en el mismo texto hacen referencia a la interacción del entorno y las personas (...) "las situaciones influyen en la gente, pero influyen en los individuos de maneras diferentes, como reconoce el enfoque interaccionista (Endler y Magnuson, 1976 citado por Cloninger, 2003: p. 203).

Estas definiciones indican que una misma situación será percibida por diferentes sujetos de diferentes formas, dependiendo de su experiencia previa, personalidad, conocimientos, etc.

Una de las teorías que mejor explica la base teórica de lo que se mide en torno al clima organizacional es la teoría de George Kelly, psicólogo norteamericano nacido en Kansas en 1905, contemporáneo de psicólogos como Abraham Maslow. La teoría que desarrolló se denomina psicología de los constructos personales.

Según esta teoría, tanto la percepción como el clima organizacional son constructos, es decir, fenómenos hipotéticos de difícil demostración ya que no son físicos; su existencia se infiere a través de manifestaciones conductuales. Ejemplos de constructos son la inteligencia y las actitudes para la psicología, y el cero para la matemática. 
La teoría de los constructos personales de Kelly se basa en "El postulado fundamental del alternativismo constructivo, el cual afirma que la gente puede interpretar cualquier evento de diversas maneras" (Endler y Magnuson, 1976 citado por Cloninger, 2003: p. 402).

\section{TÉCNICAS DE MEDICIÓN}

En todas las investigaciones del clima organizacional - laboral realizadas y cuyos resultados o técnicas se publican en artículos científicos, la investigación sigue el esquema que consta en Reinoso (2008: pp. 39-54):

A partir del estudio de la teoría de clima organizacional clásica y la realización de entrevistas con sicólogos organizacionales, se adoptan las dimensiones del clima organizacional que aparecen con mayor frecuencia en los estudios, $y$ en torno a estas se construye un instrumento que mide las brechas existentes entre las percepciones y expectativas de los individuos para cada una de ellas. Se realiza una aplicación piloto, que permite realizar análisis de confiabilidad por medio del Alfa de Cronbach, ampliamente reconocido en la investigación y en administración. Posteriormente, se realizan los análisis de validez de contenido, criterio y constructo, utilizando las herramientas estadísticas de correlación entre variables de Pearsons.

En el caso del diseño de cuestionarios, entrevistas estructuradas o semi estructuradas y aún temas para grupos focales, para medir el clima organizacional, es necesario conformar un grupo de expertos que validen las preguntas, percepciones u objetos que vamos a investigar. Para el efecto se determina el número óptimo de expertos, que está en función del tamaño de la muestra y, por lo tanto, del tamaño del universo.

Otro aspecto a considerarse es el relacionado con los criterios de inclusión de la frase, tema, objeto o percepción dentro del instrumento de investigación del clima organizacional. Entre los más utilizados están los criterios de Moriyama (Bethencourt, 2012) con el "Instructivo para el criterio de expertos sobre instrumento de recogida de datos", en donde cada uno de los criterios debe calificarse como: Nada - 0, Poco 1, Moderadamente - 2, Mucho - 3:
1. Comprensible: se refiere a si se comprende o no

2. Sensible a variaciones en el fenómeno: si usted considera que puede discriminar a individuos con los aspectos (ítems) que se evalúan, en relación con el fenómeno que se quiere medir

3. Si el instrumento (ítem) tiene suposiciones básicas justificables e intuitivamente razonables: es decir, si existe una justificación para la presencia de dicho ítem en el instrumento

4. Componentes claramente definidos: o sea, si el ítem tiene una definición clara

5. Si deriva de datos factibles de obtener: si el dato correspondiente al ítem evaluado se puede recoger

Las frases del cuestionario pueden plantearse de diversas formas; así por ejemplo, como preguntas o afirmaciones. Las preguntas pueden ser abiertas o cerradas, de elección forzosa, dicotómica o tricotómica, las respuestas también pueden plantearse en escalas de gradación Likert.

\section{Consistencia interna del instrumento de medición.-}

La consistencia interna del instrumento de medición, se mide generalmente mediante el coeficiente denominado Alfa de Cronbach (Hernández, 2013):

\begin{abstract}
Cuando se trata de instrumentos de recolección de datos, siempre es necesario saber si estos son confiables, es decir, se requiere determinar si no hay una variabilidad significativa de los resultados cuando se aplica el instrumento repetida veces. Una de las pruebas más usadas para la verificación de la confiabilidad es el "Alfa de Cronbach". Este coeficiente sirve para medir la confiabilidad de una escala de medida y fue propuesto por Cronbach en el año 1951.
\end{abstract}

La comparación por pares se utiliza en lugar del coeficiente Alpha de Cronbach para medir la consistencia interna del instrumento de medición y se aplica cuando el número de objetos, situaciones, suposiciones u observaciones a medir es pequeño (...). "En esta técnica, los objetos, por ejemplo diez, se agrupan en todas las parejas posibles (con diez objetos podemos hacer 45 parejas); después se presentan estas parejas una a la vez, y se le pide al sujeto que diga cuál estímulo tiene mayor proporción del atributo, o qué persona, por ejemplo es más diestra como maquinista" (Summers, 1976: p. 71). Si el número de observaciones, percepciones u objetos es 20 o más, esta técnica se 
vuelve inmanejable por el número elevado de pares que se debe comparar, en cuyo caso se recomienda utilizar la técnica de categorías de rango, en donde se les pide a los expertos que nos ayuden a medir la consistencia interna de cada ítem del cuestionario en un rango de uno a cinco; cinco puede ser la más alta categoría de la estructuración del estímulo, objeto o percepción y uno la más pobre. Posteriormente se obtiene la media de las respuestas de los expertos y es posible establecer las frases del cuestionario que tienen mayor validez interna.

Confianza en los datos.- Llamada comúnmente confiabilidad, hace referencia a "El grado de aproximación con el cual se mide, sin importar, aquello que se mide. Por ende, se refiere al grado en el cual las aproximaciones contendrán errores de medición únicamente, sin verse contaminadas por errores de muestreo" (Arias, 1980).

La American Psychological Asociation, establece tres índices de consistencia de los cuestionarios: consistencia interna, equivalencia y estabilidad. Índices que deberán determinarse en función de la información que se investigue.

\section{RESULTADOS Y DISCUSIÓN}

A pesar de todas las precauciones que se tome al diseñar cuestionarios para medir el clima laboral, siempre existirá, por parte del encuestado, el riesgo de que no sea sincero en sus respuestas. Esto puede deberse a varias causales, entre ellas: el afán de causar una buena impresión, el temor a represalias si contesta con la verdad, ciertas características de personalidad, falta de explicación de los fines de la investigación.

Otro aspecto que se debe considerar se refiere a la teoría que subyace al cuestionario, es decir, si en el clima organizacional se consideró como factor, por ejemplo, a la motivación y las preguntas corresponden a la teoría de factores higiénicos de Herzberg, entonces se está midiendo, solamente, una parte de la motivación en el trabajo. Similar situación ocurre con los demás factores o elementos del clima organizacional o clima laboral.

Un aspecto que merece mencionarse respecto de las mediciones del clima laboral, se relaciona con la existencia de sub climas dentro de la organización, es decir, cada departamento y aún cada sección tendrá un clima laboral, por lo que, no se debería generalizar a toda la institución los resultados globales.
Otro aspecto se refiere a que la medición del clima organizacional se contextualiza en un momento y situación determinados; factores como el cambio de oficina, despedido de empleados, cambio de jefe, motivarán cambios en el clima organizacional.

\section{CONCLUSIONES}

El clima organizacional se puede medir utilizando diversas técnicas e instrumentos, cada uno de los cuales debe regirse a normas y protocolos de investigación científica; caso contrario, los resultados e información obtenida no aportarán para optimizar y mejorar las situaciones y ambientes psicológicos, sociales y físicos en los que se desenvuelve el trabajo.

Cuando se mide el clima laboral, se miden percepciones vinculadas a constructos, emociones, sentimientos, personalidad, creencias, entre otros elementos, por lo que, la responsabilidad de las personas que elaboran los instrumentos de medición y los aplican exige un grado elevado de conocimiento y de ética profesional.

\section{REFERENCIAS BIBLIOGRÁFICAS}

Arias F. (1980). Introducción a la Técnica de investigación en ciencias de la administración y el comportamiento. México, D.F.: Editorial Trillas.

Bethencourt (2012). Exploración sobre la calidad de las investigaciones y la producción científica en Camagüey, Cuba. Revistas médicas cubanas, vol. 50 año 2012. Recuperado de http://www.bvs.sld.cu/ revistas/hie/vol50_1_12/a0108112.pdf

Carrie, S. (2012). Los beneficios de mantener un buen clima organizacional. Recuperado de http:// www.educamericas.com/articulos/reportajes/ los-beneficios-de-mantener-un-buen-climaorganizacional

Cloninger. S. (2003). Teorías de la Personalidad. México, D.F.: Editorial Prentice Hall.

Cotton P. (2013) Desarrollando un clima organizacional óptimo. Recuperado de http:// www.losrecursoshumanos.com/contenidos/2069desarrollando-un-clima-organizacional-optimo.html

Guardiola P. (2002). Mis publicaciones de sociología. Recuperado de http://www.um.es/docencia/ pguardio/documentos/percepcion.pdf

Hernández O. (2013). Cómo calcular el Coeficiente de Alfa de Cronbach, sin despeinarse. Universidad 
Nacional Rómulo Gallegos. Recuperado de http:// infounerg.net/?m=tutoriales

Reinoso H. (2008). "Diseño y Validación de un modelo de medición del clima organizacional basado en percepciones y expectativas". Revista Ingeniería Industrial, ISSN-e 0718-8307, №. 1, 2008 , págs. 39-54.

Summers, G. (1976). Medición de Actitudes. Chicago, Illinois: Editorial Trillas.

Valbuena de la Fuente F. (1979). La comunicación y sus clases, aplicaciones a diversos campos de la actividad humana. Zaragoza: Editorial Edelvives. 